\title{
Carbon Ion Radiotherapy
}

National Cancer Institute

\section{Source}

National Cancer Institute. Carbon Ion Radiotherapy. NCI Thesaurus. Code C160816.

Ion beam radiation therapy that uses charged carbon particle. Compared to proton beam therapy, the larger mass of carbon results in decreased beam scattering, yielding a sharper dose distribution border with minimal penumbra and two to three times the relative biological effect. 